\title{
Spleen assessment after laparoscopic transperitoneal left adrenalectomy: preliminary results
}

\author{
Pasquale Cianci $^{1} \cdot$ Alberto Fersini $^{1} \cdot$ Nicola Tartaglia $^{1} \cdot$ Amedeo Altamura $^{1}$. \\ Vincenzo Lizzi $^{1}$ - Luca Pio Stoppino ${ }^{1}$ Luca Macarini ${ }^{1}$ - Antonio Ambrosi ${ }^{1}$. \\ Vincenzo Neri $^{1}$
}

Received: 15 April 2015/ Accepted: 19 June 2015/Published online: 3 July 2015

(C) The Author(s) 2015. This article is published with open access at Springerlink.com

\begin{abstract}
Background Several laparoscopic approaches to the adrenal gland have been described. We prefer the lateral transabdominal approach. The aim of this study is to evaluate prospectively the presence of any anatomical and dynamic changes in the spleen after laparoscopic transperitoneal left adrenalectomy (LTLA), which can cause an increased risk of early and late complications.

Methods We have evaluated 21 patients before and 6 months after surgery in order to verify the possible presence of a wandering spleen. A clinical and instrumental follow-up [ultrasound (US), magnetic resonance (MR)] were performed. During US protocol, in supine, right lateral, and orthostatic position, the longitudinal and anteroposterior diameter of the spleen and the resistive index within $3 \mathrm{~cm}$ of the origin of the splenic artery in three different measurements averaged were measured. MR protocol evaluated, in supine and right lateral position, the splenic volume and its distances from the diaphragm dome and the lateral margin of the costal arch.

Results $p$ Values calculated for each parameter were not statistically significant. Our results confirm the absence of any anatomical and dynamic changes in the spleen after LTLA.

Conclusions The most common complications after laparoscopic adrenalectomy are well known and widely described. Our experience does not exclude the occurrence of a wandering spleen, but allows us to state that a rightful mobilization of the pancreaticosplenic block can avoid this event, and in agreement with other authors, the presence of a wandering spleen remains an isolated complication.
\end{abstract}

Pasquale Cianci

ciancidoc1@virgilio.it

1 Department of Medical and Surgical Sciences, University of Foggia, Luigi Pinto Str 1, 71122 Foggia, Italy
Keywords Laparoscopic adrenalectomy · Wandering spleen · Endocrine surgery - Minimally invasive surgery · Adrenal diseases

The laparoscopic approach for adrenalectomy was first described by Gagner [1]. Since its introduction, the technique has rapidly gained popularity among surgeons dealing with adrenal disease, and it is presently considered the "gold standard" procedure for the removal of benign and selected malignant adrenal pathologic masses even greater than $7 \mathrm{~cm}[2,3]$.

The most accepted route to the adrenal gland is the laparoscopic transperitoneal approach, with the patient placed on hyperextended lateral flank [3, 4]. On the left side, the pancreatic tail and the spleen are fully mobilized and dropped medially. A greater mobilization of the pancreaticosplenic would have recreated the conditions of a wandering spleen manifested by its complication such as gastric volvulus [5-9]. The overall complication rate associated with laparoscopic adrenalectomy was $9.5 \%$ (range 2.9-20) [3]. The most common complications after laparoscopic adrenalectomy are well known and widely described include bleeding, organ injury, wound hematoma and wound infection, systemic complication $[10,11]$. The aim of this study is to evaluate prospectively the presence of any anatomical and dynamic changes in the spleen after laparoscopic transperitoneal left adrenalectomy (LTLA), which can cause an increased risk of early and late complications.

\section{Materials and methods}

From January 2008 to July 2013, we treated 71 patients with adrenal diseases in our Department. During the same period, 43 laparoscopic procedures were performed and of these we evaluated only the 21 laparoscopic left 
adrenalectomies, 9 males $(42.86 \%)$ and 12 females $(57.14 \%)$ with a mean age of 52.05 years (range 17-73). The characteristics of the patients are shown in Table 1. Each case has been studied by our team of endocrinologists prior to intervention, and the indications for surgery were nonfunctioning adenoma $(n=6)$, Conn's syndrome $(n=4)$, pheochromocytoma $(n=6)$, nodular cortical hyperplasia $(n=4)$, and metastatic lung cancer $(n=1)$ (Table 2$)$. The exclusion criteria were bilateral adrenalectomy, previous abdominal surgery, and concomitant surgical diseases. The laparoscopic operation was performed in all instances using a transperitoneal lateral approach with the patient placed in an almost full lateral position and with the operating table broken to maximum extension to allow the space between rib cage and pelvis to open fully. After access and placement of three trocars, the first step was to incise the lienal attachments to the diaphragm along the lateral border of the spleen at the splenorenal ligament, the dissection is closed when the short gastric vessels are identified posteriorly. The spleen then falls medially under gravity. The mobilization of the splenic flexure was performed only when absolutely necessary. After informed consent we have evaluated the spleen with color Doppler ultrasound and MRI scan before and 6 months after surgery. During ultrasound (US) protocol using curvilinear variable-frequency transducer with the patients in different position (supine, right lateral, orthostatic), the longitudinal and anteroposterior diameter of the spleen and the resistive index within $3 \mathrm{~cm}$ of the origin of the splenic artery in three different measurements averaged were measured. The same radiological team performed magnetic resonance (MR) protocol using a 1.5-T unit. In supine and right lateral position were measured the splenic volume and its distances from the diaphragm dome and the lateral margin of the costal arch. Data are presented as mean values and

Table 1 Demographic characteristics of the patients
Table 2 Indications for surgery

\begin{tabular}{ll}
\hline Nonfunctioning adenoma & $6 / 21(28.57 \%)$ \\
Conn's syndrome & $4 / 21(19.05 \%)$ \\
Pheochromocytoma & $6 / 21(28.57 \%)$ \\
Nodular cortical hyperplasia & $4 / 21(19.05 \%)$ \\
Metastasis (lung cancer) & $1 / 21(4.76 \%)$ \\
\hline
\end{tabular}

analyzed using a two-tailed $t$ test for paired data (Microsoft Excel 2000, Microsoft, Redmond, WA, USA). Statistical significance was taken to be a $p$ value less than 0.05 .

\section{Results}

A total of 21 LTLA were followed during the study period. Intraoperative and postoperative outcomes are summarized in Table 3. The average operation length was $159 \mathrm{~min}$ (range 120-220), and no bilateral lesion was included. Mean size of the lesions was $2.73 \mathrm{~cm}$ (range 0.7-7). The average blood loss was $77.25 \mathrm{ml}$ (range 50-110), and no patients required transfusion. The conversion rate was $0 \%$, and the complication rate was $14.28 \%$. This last includes one case of hemoperitoneum in the immediate postoperative period, caused by bleeding from a small branch of the adrenal vein promptly resolved surgically. Postoperative courses were free of complications in 19 patients. In the remaining two patients, there was one case of atrial fibrillation and one wound infection. No mortality occurred.

During the US protocol, no significant difference in the pre- and postoperative longitudinal diameter of the spleen was observed ( $p=0.531)$. Neither anteroposterior diameter of the spleen $(p=0.394)$ nor the resistive index ( $p=0.454)$ was significantly different in these reports. Also MR outcomes showed no difference between pre- and postoperative averages of splenic volume $(p=0.849)$, distance from its lateral margin to costal arch $(p=0.428)$, and its distance from diaphragm dome $(p=0.080)$. These results confirm the absence of any anatomical and dynamic changes in the spleen after LTLA (Table 4).

\section{Discussion}

Since its introduction in 1992 [1], LA has rapidly became the procedure of choice for the surgical management of most adrenal tumors. Comparing to open adrenalectomy, laparoscopic adrenalectomy offers better clinical outcomes, lower less perioperative morbidity, shorter hospitalization, and better cosmetic results [3, 12-16]. Transabdominal and retroperitoneal approaches are most common [17]. The 
Table 3 Intraoperative and postoperative data of patients undergoing LTLA

\begin{tabular}{ll}
\hline Operative time (min) & \\
Mean (SD) & $159(36.49)$ \\
Median & 150 \\
Range & $120-220$ \\
Blood loss (ml) & \\
Mean (SD) & $77.25(16.66)$ \\
Median & 77.5 \\
Range & $50-110$ \\
Tumor size (cm) & \\
Mean (SD) & $2.73(1.53)$ \\
Median & 2.5 \\
Range & $0.7-7$ \\
Conversion rate (\%) & 0 \\
Postoperative ambulation (hours) & \\
Mean (SD) & $31.25(9.10)$ \\
Median & 27.5 \\
Range & $22-50$ \\
Postoperative hospitalization (days) & \\
Mean (SD) & $4.05(1.05)$ \\
Median & 4 \\
Range & $3-6$ \\
Perioperative complications rate $(\%)$ & 4.75 \\
Postoperative complications rate $(\%)$ & 9.50 \\
\hline
\end{tabular}

majority of surgeons perform the lateral transperitoneal access that favors excellent exposure and allows gravity to aid in the retraction of adjacent organs. The posterior retroperitoneal approach is an alternative that avoids the peritoneal cavity without mobilization of pancreas and spleen and may be helpful for patients with abdominal adhesions or patients requiring bilateral adrenalectomies; however, exposure and working space are limited, and anatomical relationship may not be as familiar. The third and less common access is an anterior transperitoneal approach, which affords the traditional view of anatomy but requires considerable effort to retract adjacent organs and maintain exposure [18]. We prefer the first way that requires less dissection, better retraction of the adjacent organs, and easy removal of the surgical specimen. We used the precocious binding of the adrenal vein, the greatest care in hemostasis and in dissection, preserving the anatomical integrity of the gland and the positioning of a tubular drainage at the end of the operation [19]. The most common complications after laparoscopic adrenalectomy are well known and widely described $[10,11]$. We have evaluated 21 patients before and 6 months after surgery in order to verify the possible presence of a wandering spleen. In addition to a clinical and ultrasonographic evaluation, we have used also a MR protocol. The literature suggests that Doppler ultrasound is used to check the spleen position and parenchymal flow [20-23]. Some authors describe CT scan as the best choice to identify this rare condition [24], although Buckley et al. [25] suggest that MR imaging may confer certain advantages because of its lack of ionizing radiation, as well as its superior tissue characterization, and it is not operator dependent. Our preliminary results confirm the absence of any anatomical and dynamic changes in the spleen after LTLA, and we have compared them to the largest series in the literature (Table 5). The authors reported were chosen based on the following criteria: (1) LTA as surgical approach, (2) three trocars used, (3) single institution experience, and (4) only adult patients evaluated.

The unique case of alteration of the spleen assessment after LTLA was described by Corcione et al. [5]. He observed a case of a 57-year-old woman who has undergone LTLA and in the third postoperative month, had an acute gastric volvulus requiring surgical open treatment. In his opinion, a probable gastrosplenic ligament laxity and an excessive mobilization of the pancreaticosplenic block can be the etiology of this singular complication. In the wake of this case, Piccoli et al. [30] have evaluated 24 patients with a clinical and ultrasonographic follow-up that showed no evidence of postoperative wandering spleen. Following the procedure described by Gagner [28], we begin the mobilization of the spleen dissecting the lienal attachment to the diaphragm along the lateral border at the lienorenal ligament. Superiorly the dissection is stopped when the short gastric vessels are identified posteriorly. After this, the spleen falls medially and this favors the complete exposure of the adrenal gland. Our experience does not exclude the

Table 4 US and MR protocol findings before and six months after surgery

\begin{tabular}{lllcc}
\hline Parameters & Protocol & Preoperative findings (mean) & Postoperative findings $($ mean $)$ & $p$ value $(<0.05)$ \\
\hline Splenic volume $\left(\mathrm{cm}^{3}\right)$ & MR & 212.2476 & 210.2857 & 0.849 \\
Spleen-lateral margin/costal arch (distance in cm) & MR & 0.64 & 0.680952 & 0.428 \\
Spleen-diaphragm dome (distance in cm) & MR & 0.62 & 0.7 & 0.080 \\
Longitudinal diameter of the spleen (cm) & US & 10.92 & 10.7 & 0.531 \\
Anteroposterior diameter of the spleen $(\mathrm{cm})$ & US & 4.9 & 4.75 & 0.394 \\
Resistive index & US & 0.612381 & 0.626 & 0.454 \\
\hline
\end{tabular}


Table 5 Outcomes of the laparoscopic transperitoneal adrenalectomy (LTA) in selected large series

\begin{tabular}{|c|c|c|c|c|c|c|}
\hline References & $\begin{array}{l}\text { No. of } \\
\text { procedures }\end{array}$ & $\begin{array}{l}\text { Operative } \\
\text { time (min) }\end{array}$ & $\begin{array}{l}\text { Conversion } \\
\text { rate }(\%)\end{array}$ & $\begin{array}{l}\text { Complication } \\
\text { rate }(\%) / \mathrm{No} \text {. of WS }\end{array}$ & $\begin{array}{l}\text { Postoperative } \\
\text { hospitalization } \\
\text { (days) }\end{array}$ & $\begin{array}{l}\text { Mortality } \\
(\%)\end{array}$ \\
\hline Henry et al. $[10]^{\mathrm{a}}$ & 169 & 129 & 5 & $7.5 / 0$ & 5.4 & 0 \\
\hline Guazzoni et al. [26] $]^{\mathrm{a}}$ & 161 & 160 & 2.5 & $5.1 / 0$ & 2.8 & 0 \\
\hline Porpiglia et al. [27] ${ }^{\mathrm{a}}$ & 125 & 139 & 3.2 & $11.2 / 0$ & 4 & 0.8 \\
\hline Gagner et al. [28] ${ }^{\mathrm{a}}$ & 100 & 123 & 3 & $12 / 0$ & 2.4 & 0 \\
\hline Conzo et al. [29] ${ }^{\mathrm{a}}$ & 88 & 137.33 & 1.13 & $5.6 / 0$ & 3.5 & 0 \\
\hline Piccoli et al. [30] ${ }^{\mathrm{b}}$ & 24 & 89 & 0 & $8 / 0$ & 2 & 0 \\
\hline Neri et al. $[19]^{\mathrm{b}}$ & 21 & 159 & 0 & $14.28 / 0$ & 4.05 & 0 \\
\hline
\end{tabular}

WS wandering spleen

a Data include laparoscopic right and left adrenalectomies, ${ }^{\mathrm{b}}$ Data include only laparoscopic left adrenalectomies

occurrence of this event, but allows us to state that a rightful mobilization of the pancreaticosplenic block can avoid the occurrence of a wandering spleen, and in agreement with other authors, the presence of a wandering spleen remains an isolated complication.

Disclosures Pasquale Cianci, Alberto Fersini, Nicola Tartaglia, Amedeo Altamura, Vincenzo Lizzi, Luca Pio Stoppino, Luca Macarini, Antonio Ambrosi, Vincenzo Neri have no conflicts of interest or financial ties to disclose.

Open Access This article is distributed under the terms of the Creative Commons Attribution 4.0 International License (http://creativecommons.org/licenses/by/4.0/), which permits unrestricted use, distribution, and reproduction in any medium, provided you give appropriate credit to the original author(s) and the source, provide a link to the Creative Commons license, and indicate if changes were made.

\section{References}

1. Gagner M, Lacroix A, Bolté E (1992) Laparoscopic adrenalectomy in Cushing's syndrome and pheochromocytoma. N Engl J Med 327(14): 1033

2. Ramacciato G, Mercantini P, La Torre M, Di Benedetto F, Ercolani G, Ravaioli M, Piccoli M, Melotti G (2008) Is laparoscopic adrenalectomy safe and effective for adrenal masses larger than $7 \mathrm{~cm}$ ? Surg Endosc 22(2):516-521 (Epub 2007 Aug 18)

3. Assalia A, Gagner M (2004) Laparoscopic adrenalectomy. Br J Surg 91(10):1259-1274

4. Lezoche E, Guerrieri M, Crosta F, Paganini A, D’Ambrosio G, Lezoche G, Campagnacci R (2008) Perioperative results of 214 laparoscopic adrenalectomies by anterior transperitoneal approach. Surg Endosc 22(2):522-526 (Epub 2007 Aug 19)

5. Corcione F, Tricarico F, Barbaros U, Marzano E, Montini F, Trombetti A (2008) Gastric volvulus after laparoscopic left adrenalectomy: case report. Surg Laparosc Endosc Percutan Tech 18(2):207-208

6. Matsushima K, Kayo M, Hachiman H, Gushimiyagi M (2006) Laparoscopic repair of gastric volvulus associated with wandering spleen in an adult: report of a case. Surg Today 36(9): 843-845
7. Tadaharu O, Rumi O, Go M, Lane Geoffrey J, Toshiaki T, Atsuyuki Y (2010) Laparoscopic splenopexy and gastropexy for wandering spleen associated with gastric volvulus. Pediatr Surg Int 26:1053-1055

8. Lianos G, Vlachos K, Papakonstantinou N, Katsios C, Baltogiannis G, Godevenos D (2013) Gastric volvulus and wandering spleen: a rare surgical emergency. Case Rep Surg 2013:561752. doi:10.1155/2013/561752 (Epub 2013 Feb 7)

9. Ooka M, Kohda E, Iizuka Y, Nagamoto M, Ishii T, Saida Y, Shimizu N, Gomi T (2013) Wandering spleen with gastric volvulus and intestinal non-rotation in an adult male patient. Acta Radiol Short Rep 2(7):2047981613499755. doi:10.1177/ 2047981613499755 (eCollection 2013)

10. Henry JF, Defechereux T, Raffaelli M, Lubrano D, Gramatica L (2000) Complications of laparoscopic adrenalectomy: results of 169 consecutive procedures. World J Surg 24(11):1342-1346

11. Ku JH, Yeo WG, Kwon TG, Kim HH (2005) Laparoscopic adrenalectomy for functioning and non-functioning adrenal tumors: analysis of surgical aspects based on histological types. Int J Urol 12(12):1015-1021

12. Brunt LM, Doherty GM, Norton JA, Soper NJ, Quasebarth MA, Moley JF (1996) Laparoscopic adrenalectomy compared to open adrenalectomy for benign adrenal neoplasms. J Am Coll Surg 183(1): $1-10$

13. Dudley NE, Harrison BJ (1999) Comparison of open posterior versus transperitoneal laparoscopic adrenalectomy. Br J Surg 86(5):656-660

14. Thompson GB, Grant CS, van Heerden JA, Schlinkert RT, Young WF Jr, Farley DR, Ilstrup DM (1997) Laparoscopic versus open posterior adrenalectomy: a case-control study of 100 patients. Surgery 122(6):1132-1136

15. Imai T, Kikumori T, Ohiwa M, Mase T, Funahashi H (1999) A case-controlled study of laparoscopic compared with open lateral adrenalectomy. Am J Surg 178(1):50-53 (discussion 54)

16. Brunt LM (2002) The positive impact of laparoscopic adrenalectomy on complications of adrenal surgery. Surg Endosc 16(2):252-257 (Epub 2001 Nov 16)

17. MacGillivray DC, Shichman SJ, Ferrer FA, Malchoff CD (1996) A comparison of open vs laparoscopic adrenalectomy. Surg Endosc 10(10):987-990

18. McKinlay R, Mastrangelo MJ Jr, Park AE (2003) Laparoscopic adrenalectomy: indications and technique. Curr Surg 60(2): 145-149

19. Neri V, Ambrosi A, Fersini A, Valentino TP (2005) Laparoscopic adrenalectomy: transperitoneal lateral approach. Cases study. Ann Ital Chir 76(2):123-126 
20. Di Crosta I, Inserra A, Gil CP, Pisani M, Ponticelli A (2009) Abdominal pain and wandering spleen in young children: the importance of an early diagnosis. J Pediatr Surg 44(7):1446-1449

21. Herneth AM, Pokieser P, Philipp MO, Bader TR, Helbich TH, Mallek R, Mostbeck GH (2001) Role of Doppler sonography in the evaluation of accessory spleens after splenectomy. J Ultrasound Med 20(12):1347-1351

22. Allen KB, Gay BB Jr, Skandalakis JE (1992) Wandering spleen: anatomic and radiologic considerations. South Med J 85(10): 976-984

23. Chen JW, Yeh DM, Peng SH, Chen GS, Tseng YH, Lin CW, Tyan YS, Tsao TF (2012) Sonographic diagnosis of a subclinical wandering spleen: role of the decubitus position. J Ultrasound Med 31(3):483-487

24. Ben Ely A, Zissin R, Copel L, Vasserman M, Hertz M, Gottlieb P, Gayer G (2006) The wandering spleen: CT findings and possible pitfalls in diagnosis. Clin Radiol 61(11):954-958

25. Buckley O, Ward EV, Doody O, Torreggiani WC (2007) MRI of the wandering spleen. Clin Radiol 62(5):504 (Epub 2007 Feb 22)
26. Guazzoni G, Cestari A, Montorsi F, Lanzi R, Nava L, Centemero A, Rigatti P (2001) Eight-year experience with transperitoneal laparoscopic adrenal surgery. J Urol 166(3):820-824

27. Porpiglia F, Garrone C, Giraudo G, Destefanis P, Fontana D, Morino M (2001) Transperitoneal laparoscopic adrenalectomy: experience in 72 procedures. J Endourol 15(3):275-279

28. Gagner M, Pomp A, Heniford BT, Pharand D, Lacroix A (1997) Laparoscopic adrenalectomy: lessons learned from 100 consecutive procedures. Ann Surg 226(3):238-246 (discussion 246-7)

29. Conzo G, Pasquali D, Della Pietra C, Napolitano S, Esposito D, Iorio S, De Bellis A, Docimo G, Ferraro F, Santini L, Sinisi A (2013) Laparoscopic adrenal surgery: ten-year experience in a single institution. BMC Surg 13(Suppl 2):S5. doi:10.1186/14712482-13-S2-S5 (Epub 2013 Oct 8)

30. Piccoli M, De Luca GM, Pasculli A, Angelini M, Guicciardi L, Mullineris B, Marchi D, Melotti G (2014) Laparoscopic transperitoneal left adrenalectomy and wandering spleen risk. JSLS 18(3). pii: e2014.00278. doi: 10.4293/JSLS.2014.00278 